NoTe

\title{
Double Parathyroid Carcinoma
}

\author{
KAORI KAMEYAMA AND HIROSHI TAKAMI* \\ Division of Diagnostic Pathology, Keio University School of Medicine, Shinjuku-ku, Tokyo 160-8582, Japan \\ *Department of Surgery, Teikyo University School of Medicine, Itabashi-ku, Tokyo 173-8610, Japan
}

\begin{abstract}
The incidence of parathyroid carcinoma is rare. We recently encountered a case of double carcinoma, located in the right and left upper parathyroid glands. A 67-year old man came to the Teikyo University Hospital because of his bilateral parathyroid masses with hypercalcemia and high parathyroid hormone (PTH). Preoperative diagnoses were parathyroid adenoma in the left lobe and papillary thyroid carcinoma or parathyroid carcinoma in the right lobe. First, the left tumor was removed; however, iPTH was still high. The right mass was then resected and iPTH fell to normal range. Histological examination revealed both tumors were parathyroid carcinoma. To the best of our knowledge, this is the first report of double parathyroid carcinoma confirmed histologically.
\end{abstract}

Key words: Parathyroid gland, Double carcinoma, Primary hyperparathyroidism, Parathyroid hormone (PTH), Quick intact PTH assay

(Endocrine Journal 50: 477-479, 2003)

THE cause of primary hyperparathyroidism is usually an adenoma or hyperplasia, while carcinoma is rare, accounting for up to $5 \%$. Moreover, the incidence of double parathyroid adenoma is less than $5 \%$ in primary hyperparathyroidism. Herein we report the first case of double parathyroid carcinoma evaluated histologically.

\section{Case Report}

A 67-year-old man was found to have bilateral parathyroid masses with hypercalcemia and high parathyroid hormone (PTH) at a local hospital, and referred to the Department of Surgery, Teikyo University Hospital for further examination. He had no family history of parathyroid disorder or multiple endocrine neoplasia syndrome. Physical examination showed no palpable neck mass. Ultrasonography demonstrated a

Received: February 17, 2003

Accepted: May 6, 2003

Correspondence to: Kaori KAMEYAMA, M.D., Division of Diagnostic Pathology, Keio University School of Medicine, 35 Shinanomachi, Shinjuku-ku, Tokyo 160-8582, Japan well-defined and homogenous mass $(20 \times 21 \times 14 \mathrm{~mm})$ behind the left thyroid, suggesting parathyroid adenoma and a heterogeneous mass $(23 \times 17 \times 20 \mathrm{~mm})$ with an ill-defined, irregular margin embedded in the right lobe, suggesting papillary carcinoma or intrathyroidal parathyroid carcinoma. A sesta technetium$99 \mathrm{~m}$ methoxyisobutylisonitrile (MIBI- ${ }^{99 \mathrm{~m}} \mathrm{Tc}$ ) subtraction scintigram revealed sestaMIBI accumulations in the left upper pole and right middle lobe of the thyroid in the delayed phase. Laboratory data showed high serum calcium ( $5.8 \mathrm{mEq} / 1$, normal range 4.2-5.2), high HS-PTH $(9100 \mathrm{pg} / \mathrm{ml}, 160-520)$, high сPTH (3.4 $\mathrm{ng} / \mathrm{ml}, 0-0.5)$, high iPTH $(270 \mathrm{pg} / \mathrm{ml}, 15-50)$, normal serum phosphorus $(2.1 \mathrm{pg} / \mathrm{dl}, 2.0-5.0)$, and high alkaline phosphatase (248 IU, 66-220). The preoperative diagnoses were parathyroid adenoma in the left lobe, and papillary carcinoma or parathyroid carcinoma in the right lobe.

Surgery was performed using intraoperative quick intact PTH assay (QPTH). The preoperative iPTH was $515 \mathrm{pg} / \mathrm{ml}(15-50)$. In the first step, an enlarged parathyroid mass was recognized at the back of the left upper lobe of the thyroid. The mass did not adhere firmly to the thyroid and was easily removed. The serum iPTH levels were still $289 \mathrm{pg} / \mathrm{ml}$ and $241 \mathrm{pg} / \mathrm{m}$ 
at 5 and 15 min after removal, respectively. At this time, the right thyroid mass was suspected to be carcinoma or adenoma of the parathyroid. Preoperative fine needle aspiration is contraindicated for parathyroid masses, to prevent dissemination of the parathyroid cells. The right lobe was resected, including the embedded intrathyroid mass. The iPTH level fell to normal range $(48 \mathrm{pg} / \mathrm{ml})$ at $15 \mathrm{~min}$ after right lobectomy. Paratracheal lymph node dissection was performed on the right side. The patient is alive and well at 8 months after surgery and his iPTH level is within normal range.

\section{Pathological finding}

The left upper parathyroid tumor had a thick fibrous capsule and invaded surrounding tissue. There was no vascular invasion. The tumor cells were separated by fibrous bands (Fig. 1). The tumor formed a trabecular or solid architecture without necrotic foci. The tumor cells were chief cells with mild polymorphism. Nucleoli were not apparent. Mitotic activity was relatively low ( $<5 / 50$ high power field).

The right upper parathyroid tumor was located within the right thyroid lobe. The tumor was a cystic mass with a thick fibrous capsule. The tumor cells invaded the thyroid tissue with hemosiderin deposition, and replaced thyroid follicles (Fig. 2). No vascular invasion was seen. Necrotic foci were not evident. Nucleoli were not apparent. The tumor proliferated as a solid mass composed of chief cells with moderate polymorphism. Mitoses were virtually absent.

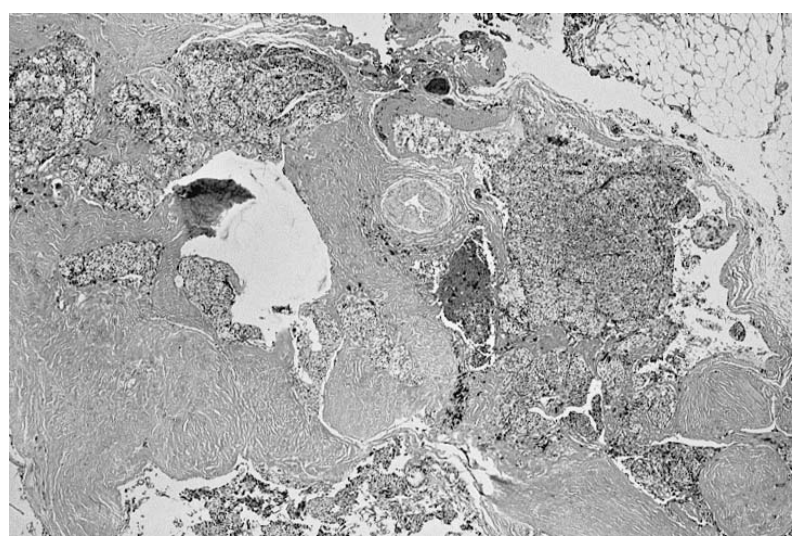

Fig. 1. Microscopic appearance of the left upper parathyroid tumor. Tumor cells with fibrous bands proliferate (Hematoxylin-Eosin stain, $\times 40$ ).
The right lower parathyroid gland showed mild atrophic change. There were no lymph nodal metastases.

\section{Discussion}

In a larger review of parathyroid carcinoma in several institutions, the incidence was $0.3 \%-5.6 \%[1-5]$ in patients with primary hyperparathyroidism.

The diagnosis of parathyroid carcinoma is often difficult to make. The histological criteria of Schantz and Castleman [6] are generally accepted, that is, the presence of a trabecular growth pattern, mitotic figures, and capsular or vascular invasion or the presence of nodal or distant metastasis. In this case, the left upper tumor showed a trabecular pattern and invasive growth, whereas the right upper tumor showed invasion to the thyroid. Adapting the criteria, these two features were taken as unequivocal evidence of parathyroid carcinoma. The criteria, presented by Bondeson et al. [7], also included histological features of clinically aggressive carcinomas having the features of macronucleoli, necrosis and mitotic activity $(>5 / 50$ hpf). On the other hand, clinically, this patient was in Stage I (T1NOM0), based on the staging system proposed by Shaha and Shah [8]. According to these histological and clinical studies of the outcome of parathyroid carcinoma, these two tumors may both have a favorable clinical course.

To the best of our knowledge, this is the first case of double parathyroid carcinoma evaluated histologically.

Double adenoma is rare. According to Attie et al.

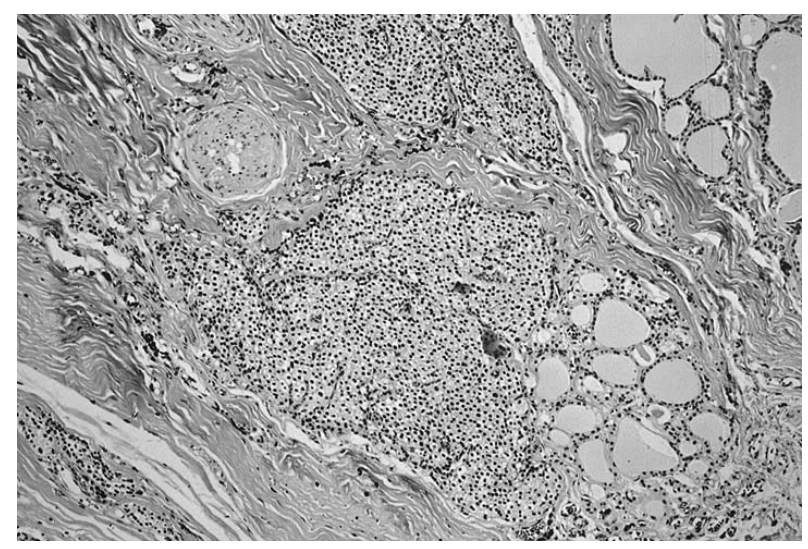

Fig. 2. Microscopic appearance of the right upper parathyroid tumor. Tumor cells invade the surrounding thyroid tissue (Hematoxylin-Eosin stain, $\times 100$ ). 
[9], multiple enlarged parathyroid glands have been reported in $1.7 \%$ to $8.5 \%$ of primary hyperparathyroidism cases. However, as they mentioned, a controversy remains as to whether this represents adenomas entirely. Indeed, the incidence of multiple adenomas has decreased over the years, and this might reflect awareness of increasing misdiagnosis of primary chief cell hyperplasia. In recent years, the incidence of double adenoma has been thought to be less than $5 \%$ in primary hyperparathyroidism. Our case is true double neoplastic lesion according to the clinical and histological criteria of Harness et al. [10].

Our case indicates that QPTH is the useful tool for detecting double parathyroid tumors even if one is located in the thyroid gland. Moreover, although some investigators have stated that the existence of double adenoma is questionable, our case confirms that two such neoplastic lesions can occur in the same patient.

\section{References}

1. Sandelin K, Thompson NW, Bondeson L (1991) Metastatic parathyroid carcinoma: Dilemmas in management. Surgery 110: 978-986.

2. Wynne AG, van Heerden J, Carney JA, Fitzpatrick LA (1992) Parathyroid carcinoma: clinical and pathological features in 43 patients. Medicine 71: 197-205.

3. Cohn K, Silverman M, Corrado J, Sedgewick C (1985) Parathyroid carcinoma: The Lahey Clinic experience. Surgery 98: 1095-1110.

4. Fujimoto Y, Obara T, Ito Y, Kanazawa K, Aiyoshi Y, Nobori M (1984) Surgical treatment of ten cases of parathyroid carcinoma: importance of an initial en bloc tumor resection. World J Surg 8: 392-400.

5. Shortell CK, Andrus CH, Phillips CE Jr, Schwartz SI (1991) Carcinoma of the parathyroid gland: a 30-year experience. Surgery 110: 704-708.

6. Schantz A, Castleman B (1973) Parathyroid carcinoma: A study of 70 cases. Cancer 31: 600-605.

7. Bondeson L, Sandelin K, Grimelius L (1993) Histopathological variables and DNA cytometry in parathyroid carcinoma. Am J Surg Pathol 17: 820-829.

8. Shaha AR, Shah JP (1999) Parathyroid carcinoma: a diagnostic and therapeutic challenge. Cancer 86: 378380.

9. Attie JN, Bock G, Auguste LJ (1990) Multiple parathyroid adenomas: report of thirty-three cases. Surgery 108: 1014-1019.

10. Harness JK, Ramsburg SR, Nishiyama RH, Thompson NW (1979) Multipla adenomas of the parathyroid: do they exist? Arch Surg 114: 468-474. 